\title{
INÍCIO DE CRISES EPILÉPTICAS NA MENARCA
}

\author{
DIOSELY C. SILVEIRA* - CARLOS A.M. GUERREIRO**
}

\begin{abstract}
RESUMO - Nove pacientes com início das crises epilépticas coincidindo com a menarca foram avaliadas. Duas tinham crises generalizadas e 7 crises com início parcial. 0 exame físico e neurológico foram normais em todas, exceto em uma que tinha estenose aórtica. O EEG mostrou ondas agudas focais nas regiões temporais em 4 pacientes, ondas lentas intermitentes generalizadas em uma e foi normal nas outras 4. Sete das 9 pacientes que tiveram a primeira crise na menarca apresentavam exacerbação das crises epilépticas no período peri-ᄀ menstrual, o que poderia sugerir estarem estas pacientes mais sujeitas a aumento na frequência das crises nesse período em ciclos subsequentes. Este achado necessita de estudo mais amplo para confirmação e pode contribuir para melhor orientação terapêutica nessas mulheres.
\end{abstract}

Epileptic seizures starting in menaxehe

SUMMARY - Nine patient whose epileptic seizures had began in the menarche phase were studied. Two of these patients had generalized seizures and seven partial seizures with or without generalization. The physical and neurologic exam was normal in all patients except one who had aortic stenosis* The EECr showed focal spikes in temporal regions in four patients, intermitent generalized slow waves in one and was normal in four patients. Seven of these patients complained of increasing of the seizure frequency near to the menstrual period. Data registered are discussed. It is concluded that the observation of a larger number of patients is necessary to confirm these data.

Em 1885, Gowers relatou tendência de iniciar-se a epilepsia durante a puberdade 5. Turner, em 1907, também levantou esta questão 27. Mais tarde, Bander et al.2 notaram que 4 de 29 mulheres com crises catameniais tinham tido inicio das crises na menarca. Logothetis et al. ${ }^{16}$ observaram que o início das crises ocorreu dentro dos três primeiros anos da menarca em 16 de 25 pacientes com exacerbação catamenial. Lennox e Lennoxi* encontraram associação entre a primeira crise e o primeiro ano da menarca em $25 \%$ de 387 mulheres.

Apresentamos 9 pacientes epilépticas crônicas cujas crises se iniciaram concomitantemente ao período da menarca e discutimos a possível fisiopatologia à luz dos dados disponíveis.

\section{CASUÍSTICA E RESULTADo}

Nove pacientes do Ambulatório de Epilepsia do Hospital das Clínicas da UNICAMP, com idades entre 20 e 44 anos e cujas crises epilépticas coincidiram com o período da menarca, sâo estudadas. Todas foram submetidas a anamnese detalhada, exame físico, exame neurológico e eletrencefalograma (EECr). A tomografia computadorizada craniana (TCC) foi realizada em três pacientes e o líquido cefalorraquidiano (JJJR) em 7. A presença ou náo de piora perimenstrual das crises epilépticas foi avaliada pela anamnese em três pacientes, e por um calendário: por período inferior a 6 meses em duas; por período entre 6 meses e 2 anos em quatro pacientes.

Os resultados são mostrados na Tabela 1. A menarca ocorreu entre 9 e 15 anos. Os tipos de crises foram variáveis, sendo generalizadas em duas pacientes e parciais com ou

Departamento de Neurologia, Faculdade de Ciências Médicas, Universidade Estadual de Campinas (UNICAMP): * Professor Assistente; ** Professor Assistente Doutor.

Dr. Carlos Alberto Mantovani Guerreiro - Departamento de Neurologia, Faculdade de Ciências Médicas, UNICAMP - Caixa Postal 6111 - 13081 Campinas SP - Brasil. 
sem generalização secundária nas demais. Duas pacientes tinham suas crises controladas por mais de um ano, quatro tinham crises uma a três vezes por mês e três tinham crises uma a auatro vezes por semana.

O exame físico e neurológico foi normal em todias as pacientes, exceto em uma que apresentava estenose aórtica. O EEG mostrava ondas agudas focais na região temporal direita em três pacientes, na região temporal esquerda e direita em uma, ondas lentas intermitentes generalizadas em uma, e foi normal nas quatro pacientes restantes. A TOC foi normal em duas pacientes e mostrou leve atrofia cortical em uma. $O$ LiCR foi normal em todas as pacientes. Das 9 pacientes, 7 apresentavam piora perimenstrual das crises.

\begin{tabular}{|c|c|c|c|c|c|c|c|}
\hline Caso & $\begin{array}{l}\text { Idade } \\
\text { (anos) }\end{array}$ & $\begin{array}{l}\text { Menarca } \\
\text { (anos) }\end{array}$ & a Tipo de crises & Frequência & Medicamentos & FEG & PPM \\
\hline 1 & 27 & 14 & CPS e CTCG & Controlada há 4 anos & CZP 600 & Normal & $\mathbf{N}$ \\
\hline 2 & 37 & 14 & CTCG & Controlada há 2 anos & DPH 300 & OLIG & $\mathbf{s}$ \\
\hline 3 & 35 & 12 & CM e CTCG & 2 vezes $/$ mês & VS 1000 & Normal & $\mathbf{S}$ \\
\hline 4 & 28 & $\mathbf{1 5}$ & CPS, CPC, CTCG & $1 \mathrm{vez} / \mathrm{mês}$ & CZP 1400 & Normal & $\mathbf{N}$ \\
\hline $\mathbf{5}$ & 36 & 12 & CPC, CTCG & 2 vezes/mês & CZP 600 & OARTD & $\mathbf{S}$ \\
\hline 6 & 25 & 9 & CPC, CTCG & 2 vezes/mês & CZP 1200 & OARTEED & $\mathbf{S}$ \\
\hline 7 & 28 & 10 & CPC, CTCG & $1 \mathrm{vez} /$ semana & CZP 1200 & OARTD & $\mathbf{S}$ \\
\hline 8 & 44 & 13 & CPS, CPC, CTCG & 1 vez/mês & DPH $\quad 400$ & OARTD & $\mathbf{S}$ \\
\hline 9 & 20 & 13 & CPC & $4 \mathrm{vez} /$ semana & CZP 800 & Normal & $\mathbf{S}$ \\
\hline
\end{tabular}

Tabela 1 - Dados referentes a pacientes com inicio das crises na menarca.

CPS, crises parciais simples; CPC, crises parciais complexas; CTCG, crises tónico-clonicas generalizadas; CZP, carbamazepina; DHP, difenil-hidantoina; VS, valproato de s6dio; OLIG, ondas lentas, intermitentes generalizadas; OARTDeE, ondas agudas região temporal esquerda/ direitia; PPM, piora perimenstrual; $N$, não; $S$, sim.

\section{COMENTÁRIOS}

Há poucos relatos sobre a influência da menarca nas crises epilépticas. Alguns pesquisadores 8.24 relataram 0 aparecimento de crises catameniais na puberdade. Rosciszewska et al.23 estudaram o efeito da menarca em 62 pacientes: em 19 não houve alteração na frequência das crises, em 20 houve aumento e em 20 houve diminuição da frequência. Observamos que 5 das 7 pacientes com CPC tiveram aumento da frequência das crises na menarca. Das 9 pacientes que apresentamos, 7 tiveram piora perimenstrual das crises $e$, uma das duas que não relatavam esta piora, tinha suas crises controladas há quatro anos. Portanto, esse achado sugere que essas pacientes são mais susceptíveis a crises epilépticas no período perimenstrual.

Tem sido sugerida etiologia hormonal para explicar o aumento cíclico das crises epilépticas. E sabido que em meninas, a partir de aproximadamente 6 anos de idade, vai ocorrendo aumento de estrógeno plasmático devido ao desenvolvimento folicular. A capacidade de produção de estrógeno pelos ovários é progressiva até o início da seleção folicular, quando é iniciado o processo ovulatório e o folículo torna-se corpo lúteo, ocorrendo a menarca. O progressivo aumento dos níveis estrogênicos pode ser um dos fatores envolvidos na fisiopatologia do início das crises na menarca. Em vários estudos o estrógeno tem mostrado efeito epileptogênico. Assim, a injeção endovenosa de estrógeno revelou acentuação da atividade epileptiforme no EEG de 11 dentre 16 mulheres epilépticas 15. Acentuação das crises em mulheres com exacerbação catamenial foi notada quando lhes era administrado estrógeno ${ }^{16}$. Por outro laḑo, a infusão endovenosa de progesterona provocou diminuição significativa no número de espículas no EEG de 4 entre 7 pacientes epilépticas'. Além disso, grande número de modelos experimentais de epilepsias, utilizando diversas espécies de animais, mostrou que o estrógeno aumenta e a progesterona diminui a susceptibilidade a crises epilépticas 3,4,6,7,9-11,13,15-17,19,21,22,25,26,28-30.

\section{REFERENCIAS}

1. Backstrom $\mathrm{T}$, Zetterlund B, Blom S, Romano M. Effects of intravenous progesterone infusions on the epileptic discharge frequency in women with parcial epilepsy. Acta Neurol Scand 1984, 69 :240-248. 
2. Bander B, Kaufman C, Dykens JW, Schleifer M, Shapiro LN. Seizures and the menstrual cycle. Am J Psychiat 1957, 113:704-708.

3. Costa PJ, Bonnycastle DD. The effect of DCA compound E, testosterone, progesterone and ACTH in modifying «agene-induced» convulsions in dogs. Arch Int Pharmacodyn 1952, $91: 330-338$.

4. Craig CR. Anticonvulsant activity of steroids: separability of anticonvulsant from hormonal effects. J Pharmacol Exp Ther 1966, 153 : 337-343.

5. Gowers WR. Epilepsy and Other Chronic Convulsive Disease: Their Causes, Symptons and Treatment. New York: William Wood, 1885.

6. Hardy RW. Unit activity in premarin-induced cortical epileptogenic foci. Epilepsia 1970, $11: 179-186$

7. Heim LM. Effect of estradiol on brain maturation: dose and time response relationships. Endocrinology 1966, $78: 1130-1134$.

8. Helmchen H, Kunkel H, Selbach H. Periodische Einflusse auf die individuale Haufigkeit cerebraler Anfalle. Arch Psychiat Nervenkr 1964, 206 : 293-308.

9. Holmes GL, Weber DA. The effect of progesterone on kindling: a developmental study. Dev Brain Res 1984, 16:45-53.

10. Hom AC, Buterbaugh GG. Estrogen alters the acquisition of seizures kindled by repeated amygdala stimulation or pentylenetetrazol administration in ovariectomized female rats. Epilepsia 1986, 27 : 103-108.

11. Julien RM, Fowler GW, Danielson MG. The effects of antiepileptic drugs on estrogen-ר -induced electrographic spike-wave discharge. J Pharmacol Exp Ther 1975, 193 : 647-656.

12. Landgren S, Backstrom T, Kalistratov G. The effect of progesterone on the spontaneous interictal spike evoked by the application of penicillin to the cats cerebral cortex. J Neurol Sci 1978, 36 : 119-133.

13. Lange SC, Julien RM. Re-evoluation of estrogen: induced cortical and thalamic paroxysmal EEG activity in the cat. Electroenceph Clin Neurophisiol 1978, 44 : 94-103.

14. Lennox WG, Lennox MA. Epilepsy and Related Disorders. Boston: Little Brown, 1960, Vol 2, p 645-650.

15. Logothetis J, Harner R. Electrocortical activation by estrogens. Arch Neurol 1960, 3: 290-297.

16. Logothetis J, Harner R, Morrel F, Torres F. The role of estrogens in catamenial exacerbation of epilepsy. Neurology 1950, $9: 352-360$.

17. Marcus EM, Watson CW, Goldman PL. Effects of steroids on cerebral electrical activity: epileptogenic effects of conjugated estrogens and related compounds in the cat and rabbit. Arch Neurol 1966, 15 : 521-532.

18. Marcus MM, Watson CW, Simin SA. Behavioral correlates of acute bilateral symmetrical epileptogenic foci in monkey cerebral cortex. Brain Res 1968, 9 : 370-373.

19. Mirsky AF, Bloch S, Tecce JJ, Lessell S, Marcus E. Visual evoked potentials during experimentally induced spike-wave activity in monkeys. Electroenceph Clin Neurophisiol $1973,35: 25-37$.

20. Newmark ME, Penry JK. Catamenial epilepsy : a review: Epilepsia 1980, 21 : 281-300.

21. Nicoletti F, Meek JL. Estradiol benzoate decreases nigral GABAergic activity in male rats. Brais Res 1985, 32 : 173-179.

22. Nicoletti F, Speciale C, Scortino MA, Summa G, Caruso GL, Patti F, Canonico PL. Comparative effects of estradiol benzoate, the antiestrogen clomiphene citrate, and the progestin in medroxyprogesterone acetate on kainic acid-induced seizures in male and female rats. Epilepsia 1985, $26: 252-257$.

23. Roscizewska D, Buntner B, Guz I, Zawisza L. Ovarian hormones, anticonvulsants drugs, and seizures during the menstrual cycle in women with epilepsy. $J$ Neurol Neurosurg Psychiat 1986, $49: 47-51$.

24. Sanches Longo LP, Gonzalez Saldaña LE. Hormones and their influence in epilepsy. Acta Neurol Lat Am 1966, 12 : 29-47.

25. Spiegel E, Wycis H. Anticonvulsant effects of steroids. J Lab Clin Med 1945, 36 : 947-953.

26. Stitt SL, Kinnard WJ. The effect of certain progestins and estrogens on the threshold of electrically induced seizure patterns. Neurology 1968, $18: 213-216$.

27. Turner WA. Epilepsy: a Study of the Idiophatic Diseasse. London: MacMillan, 1907. Reprint, New York: Raven Press, 1973.

28. Werboff J, Corcoran JB. Effects of sex hormone manipulation on audiogenic seizures. Am J Psysiol 1961, $201: 830-832$.

29. Werboff J, Hedlund L, Havlena J. Audiogenic seizures in adult male castrated rats treated with various hormones. Gen Comp Endocrinol 1963, 3 : 389-397.

30. Woolley DE, Timiras PS. The gonad-brains relationship: effects of female sex hormones on electroshock convulsion in the rat. Endocrinology 1962, $70: 196-209$. 